\title{
la filosofía con niños como experiencia transformadora. una propuesta en organizaciones sin ánimo de lucro ${ }^{1}$
}

\author{
josé barrientos-rastrojo ${ }^{2}$ \\ universidad de sevilla, espanha \\ orcid id: https:/ / orcid.org/0000-0002-0127-2644
}

\section{resumen}

Son evidentes los beneficios que supone la implementación de la metodología de Filosofía en Niños; sin embargo, un énfasis excesivo en las habilidades de pensamiento crítico provoca debilidades en su aplicación en grupos en riesgos de exclusión social. Éstos precisan una transformación profunda y no sólo una mejor forma de pensar. Esto es debido a la disonancia cognitiva provocada cuando sólo se trabaja con dimensiones discursivas: los participantes de las sesiones pueden aprender a dar buenas respuestas sin que esto se materialice más allá de las reuniones filosóficas. Un modo de enfrentar esta limitación consiste en diseñar una razón experiencial, inscrita, parcialmente, en el pensamiento cuidadoso de Mathew Lipman (2003, PP. 261-270). Si la experiencia de vida determina la construcción identitaria de los niños (Romano, 2012; Nishida, 1995; Zambrano, 1995), es crucial indagar en su significado y en cómo se articula. Por tanto, una Filosofía con Niños Experiencial, foco de este trabajo, no se centra exclusivamente en el trabajo discursivo arraigado en el pensamiento crítico. De hecho, se funda en tres pilares: (1) la creación de ejercicios experienciales, (2) el fomento de disposiciones que incentive la capacidad transformadora de éstos y (3) el diseño de escenarios y metáforas experienciales que fomenten la consecución de las verdades transformadoras. Este trabajo describe las limitaciones del abordaje cognitivo del trabajo filosófico con niños, resume las bases de la experiencialidad y añade un breve anexo del trabajo emprendido en la Universidad de Sevilla en algunas Asociaciones sin ánimo de lucro como el Proyecto Maparra de Cáritas.

palabras clave: experiencia; filosofía para/con niños; lipman; romano; zambrano.

$$
\begin{gathered}
\text { philosophy with children as a transforming experience. a proposal in non-profit } \\
\text { organizations }
\end{gathered}
$$

abstract

The benefits of implementing the methodology of Philosophy for/with Children are clear. Nevertheless, an excessive emphasis on critical thinking skills weakness its application in groups at risk of social exclusion. These groups require a profound transformation and not just a better way of thinking. This is due to the cognitive dissonance provoked when one only works with discourse: session participants can learn to give good answers without becoming able to apply them outside of philosophical settings. One way to address this limitation is to design an experiential reason, something described, in part, by Mathew Lipman (2003, pp. 261-270). If life experience determines children's identity construction (Romano, 2012; Nishida, 1995; Zambrano, 1995), it is crucial to investigate its meaning and how it is articulated. Therefore, an Experiential Philosophy with Children, the topic of this paper, does not focus exclusively on the discursive work rooted in critical

\footnotetext{
1 Este trabajo es resultado del Proyecto de Innovación Docente "La experiencia de vida proporcionada por la defensa oral de los trabajos de investigación de fin de titulación" dirigido por José Barrientos (Universidad de Sevilla, 2017-2018).

2 E-mail: barrientos@us.es
} 
la filosofía con niños como experiencia transformadora. una propuesta en organizaciones sin ánimo de lucro

thinking. Rather, it is based on three pillars: (1) the creation of experiential exercises, (2) the promotion of provisions that encourage the transformative capacity of these exercises, and (3) the design of experiential scenarios and metaphors that promote the attainment of transformative truths. This article describes the limitations of the cognitive approach of philosophical work with children, summarizes the bases of experientiality, and adds a brief appendix on the work done at the University of Seville in non-profit associations like the Maparra Project of Caritas.

keywords: life experience; philosophy for/with children; lipman; romano; zambrano.

\section{a filosofia com crianças como experiência transformadora. uma proposta em organizações sem fins lucrativos}

\section{resumo}

Os benefícios da implementação da metodologia de Filosofia com/para Crianças são evidentes; no entanto, uma ênfase excessiva nas habilidades de pensamento crítico provoca fragilidades em sua aplicação em grupos em risco de exclusão social. Essas pessoas requerem uma transformação profunda, e não apenas uma maneira melhor de pensar. Este problema se deve à dissonância cognitiva provocada quando se trabalha apenas com dimensões discursivas: os participantes das sessões podem aprender a dar boas respostas sem que isso se materialize fora dos encontros filosóficos. Uma maneira de lidar com essa limitação é projetar uma razão experiencial, parcialmente inscrita no pensamento cuidadoso de Mathew Lipman (2003, pp. 261-270). Se a experiência da vida determina a construção identitária das crianças (Romano, 2012; Nishida, 1995; Zambrano, 1995), é crucial investigar seu significado e como ela se articula. Portanto, uma Filosofia Experiencial com Crianças, foco deste artigo, não se concentra exclusivamente no trabalho discursivo enraizado no pensamento crítico. Ela é baseada em três pilares: (1) a criação de exercícios experimentais, (2) a promoção de disposições que incentivem o poder transformador destes e (3) o desenho de cenários e metáforas experimentais que promovam a obtenção de verdades transformadoras. Este artigo descreve as limitações da abordagem cognitiva do trabalho filosófico com crianças, resume as bases da experiencialidade e acrescenta um breve anexo sobre o trabalho realizado na Universidade de Sevilha em algumas associações sem fins lucrativos, tal como o Projeto Maparra de Cáritas.

palavras-chave: experiência da vida; filosofia para/com crianças; lipman; romano; zambrano. 
la filosofía con niños como experiencia transformadora. una propuesta en organizaciones sin ánimo de lucro 3

\section{1. la filosofía para niños: trascendiendo las tendencias analiticas.}

\section{1. aproximaciones críticas y discursivas de la filosofía para/con niños.}

El pensamiento crítico ha sido el más estudiado y practicado en la Filosofía con/para Niños de la clásica tríada lipmaniana (pensamiento crítico, creativo y cuidadoso). Esto ha quedado materializado en el extracto de habilidades que muchos autores proponen desarrollar en las sesiones ${ }^{4}$.

De acuerdo con los estadounidenses Lipman, Sharp y Oscanyan (1998), las potencias que se incentiva en el aula son las siguientes: mejora de la capacidad de razonar (LIPMAN, SHARP, OSCANYAN, 1998: 129-141), desarrollo de la creatividad (LIPMAN, SHARP, OSCANYAN, 1998: 143-144), descubrimiento de alternativas (LIPMAN, Sharp, Oscanyan, 1998: 148-150), de la imparcialidad (LIPMAN, SHARP, OSCANYAN, 1998: 150-152) y la coherencia (LIPMAN, SHARP, OSCANYAN, 1998: 152-155), desvelamiento de razones a favor de creencias (LIPMAN, SHARP, OSCANYAN, 1998: 155-157), estudio de las relaciones parte-todo (LIPMAN, SHARP, OSCANYAN, 1998: 161-166, 280-282), planteamiento de preguntas y respuestas eficaces y reflexionadas (LIPMAN, SHARP, OSCANYAN, 1998: 181-188; LIPMAN, 1988:62), clarificación y reformulación de afirmaciones (LIPMAN, SHARP, OSCANYAN, 1998: 209-211), inferencia de implicaciones lógicas (LIPMAN, SHARP, OSCANYAN, 1998: 202213), búsqueda de la coherencia (LIPMAN, SHARP, OSCANYAN, 1998: 214-215), petición de definiciones (LIPMAN, SHARP, OSCANYAN, 1998: 215-216), desciframiento de presuposiciones/asunciones (LIPMAN, SHARP, Oscanyan, 1998: 216-217), reconocimiento de falacias (LIPMAN, Sharp, OSCANYAN, 1998: 217-218), análisis de alternativas (LIPMAN, SHARP, OSCANYAN, 1998: 222-223), agrupamiento de ideas (LIPMAN, SHARP, OSCANYAN, 1998: 226), definiciones

\footnotetext{
3 Este trabajo es resultado del Proyecto de Innovación Docente "La experiencia de vida proporcionada por la defensa oral de los trabajos de investigación de fin de titulación" dirigido por José Barrientos (Universidad de Sevilla, 2017-2018).

${ }^{4}$ Una explicación más pormenorizada puede consultarse en BARRIENTOS-RASTROJO, 2016: 519542.
} 
la filosofía con niños como experiencia transformadora. una propuesta en organizaciones sin ánimo de lucro

y realización de actividades como concebir, asumir, hacer hipótesis (LIPMAN, 1988: 26).

Por su parte, el francés Oscar Brenifier apunta a habilidades como "proponer conceptos e hipótesis", "estructurar, articular y clarificar las ideas", "comprender las ideas de los demás y las de uno mismo", "analizar", "reformular o modificar una idea", "trabajar la relación entre el ejemplo y la idea", "argumentar", "practicar la interrogación y la objeción", desarrollar la "relación entre los conceptos, la coherencia y la legitimidad de las ideas" y el juicio, "utilizar y crear instrumentos conceptuales: error, mentira, verdad, absurdo, identidad, contrarios, categorías", "singularizar y universalizar el pensamiento", “interrogarse, descubrir y reconocer el error y la incoherencia en uno mismo" y “comprender, aceptar y aplicar las reglas de funcionamiento" (BRENIFIER, 2011: 132-133; 2012: 12-13).

El mexicano Eugenio Echeverría ha subrayado, también, la importancia de esta capacitación discursiva recalcando las siguientes: "dar razones", "distinguir las buenas razones de las malas", "construir inferencias y evaluar argumentos", "generalizar y usar analogías", “identificar, cuestionar y justificar supuestos", "reconocer contradicciones", "detectar falacias", "procurar la consistencia”, "hacer distinciones y conexiones (parte/todo, medios/fines, causa/efecto)", "hacer preguntas y hallar problemas", "hacer predicciones, formular y probar hipótesis", "proponer ejemplos y contraejemplos" y “detectar vaguedades y ambigüedades" (ECHEVERRÍA, 2006:155).

La española Carla Carreras suscribe el modelo lipmaniano (LIPMAN, 2003: 178-186) al indicar que las habilidades de pensamiento crítico son cuatro: de investigación, conceptualización, razonamiento y traducción (CARRERA, 2013: 99). Estas integran actividades como las señaladas arriba, por ejemplo, formular hipótesis, formulas conceptos precisos, poner ejemplos y contraejemplos, buscar y dar razones, hacer inferencias, razonar analógicamente, establecer relaciones de causa y efecto o relaciones entre las partes y el todo o interpretar, entre otras.

Sánchez Alcón, también español, ha aplicado la metodología lipmaniana en grupos con discapacidad intelectual. Los bloques de actuación en los encuentros 
son cuatro: pensar, o desarrollar capacidades cognitivas; sentir, o fomentar la educación emocional; educación en valores y filosofar o cimentar la dimensión metafísica (SÁNCHEZ ALCÓN, 2011: 34-35). Su metodología reproduce la de la novelas del modelo lipmaniano por lo que consiste en facilitar una reflexión en la comunidad de investigación.

De acuerdo con la portuguesa Dina Mendonça, las sesiones sirven para "clarificar cuestiones", "revisar creencias", "generar nuevas hipótesis", "saber pedir ejemplos", "clarificar afirmaciones", "identificar contradicciones", entre otras (MENDONÇA, 2011: 21). De hecho, las preguntas propuestas para las sesiones se centran en tres objetivos: pedir razones, explicaciones y clarificaciones, desarrollar otros puntos de vista y explorar el asunto (MENDONÇA, 2011: 26-27).

La brasileña Angélica Sátiro y la catalana Irene de Puig reiteran la cuádruple división de las habilidades realizada por su compañera Carreras: habilidades de investigación, conceptualización, traducción y razonamiento (DE PUIG-SÁTIRO, 2008: 67-232), siguiendo la propuesta lipmaniana. Al haber trabajado las tres en el Grupo Iref, se percibe la confluencia en sus perspectivas. El modelo cognitivo se repite en las primeras novelas de su programa Noria, aunque los últimos años le han conducido a centrarse primero en el pensamiento creativo y, posteriormente, en prácticas más dinámicas como el jardín de la mariquita Juanita.

La inglesa Ellen Duthie es la última incursión en el desarrollo del pensamiento crítico a través de su Filosofía visual. Su material, muy recomendable para el trabajo con educación infantil, se basa en un conjunto de cajas con fichas de dibujos elocuentes para el desarrollo del pensamiento crítico y discursivo. Es más, a la espalda de cada ficha, aparece una decena de preguntas para incentivar la discusión. Por último, cada caja incluye instrucciones con ejercicios y actividades para desarrollar en clase o en casa. 
la filosofía con niños como experiencia transformadora. una propuesta en organizaciones sin ánimo de lucro

\section{2. concreción de las dimensiones creativas y cuidadosas.}

Quizás sea el argentino Walter Kohan quien, desde sus comienzos, ha buscado modelos de racionalidad alternativos a la analítica ${ }^{5}$. Sin embargo, no olvida que, también, el pensamiento discursivo es esencial en el trabajo de Filosofía con Niños. En consecuencia, subraya que los encuentros deberían asegurar objetivos como "someter los puntos de vista de los otros participantes a la investigación conjunta; dar razones para apoyar los puntos de vista de los demás aun cuando se esté en desacuerdo con ellos; (...) estar abierto a cambiar los propios puntos de vista y prioridades en relación a los puntos de vista de los otros integrantes de la comunidad" (KOHAN-WAKSMAN, 2000: 23).

Indicar que todos estos autores se restringen a un abordaje analíticodiscursivo sería errar. En primer lugar, Walter Kohan nos recuerda “cuidar no sólo los procedimientos lógicos sino el crecimiento de los otros miembros de la comunidad" (KOHAN-WAKSMAN, 2000: 23). De hecho, la dimensión biográfica ${ }^{6}$ que viola los excesos formales se aprecia en diversas obras del autor, que aquí no podemos reseñar pero que recomendamos enfáticamente (KOHAN, 2015; 2016).

La dimensión social es la primera que muchas de estos autores enfatizan siguiendo el espíritu de la necesidad de la comunidad de investigación creada por Peirce. Uno de los bloques de Oscar Brenifier anima a "pensar con los otros" e integra habilidades como escucharlos, proporcionales su espacio, respetarlos, interesarse por sus pensamientos, pensar en lugar de competir con los demás (BRENIFIER, 2001: 44). Echeverría también se une a esta línea social cuando insta a "saber escuchar a los demás", "respetar a las personas y sus puntos de vista" o "discutir con base en los intereses comunes" (ECHEVERRÍA, 2006: 155). El resto de los autores citados, y otros de orientación lipmaniana, tienen clara conciencia de que el escenario más oportuno para el desarrollo del trabajo exige la

\footnotetext{
5 Bajo el concepto analítico, entendemos el trabajo discursivo realizado en las habituales sesiones de Filosofía par Niños cuya principal herramienta es el discurso reflexionado de los niños. Esto es diferente a si se realizasen talleres donde se intentasen desvelar el significado de un texto a través de un trabajo con la corporalidad (apelando a Merleau-Ponty) o a un desvelamiento de verdades a partir de experiencias de vidas mediadas por ejercicios propios de los estoicos. Las limitaciones de este artículo no permiten ampliar más este asunto, pero puede profundizarse en el tema en BARRIENTOS-RASTROJO, 2015; 2016.

${ }^{6}$ Amplíese este punto en BARRIENTOS-RASTROJO, 2013.
} 
constitución de una sociedad democrática por lo que deben tener presente la necesidad social en el programa.

La dimensión creativa constituye un segundo brazo crucial. El pensamiento creativo el segundo de los tres tipos citados por Lipman (LIPMAN, 2003: 243-260). Sátiro es una de sus más pujantes defensoras (DE PUIG-SÁTIRO, 2008: 37-47) tanto por su formación en procesos de creatividad como por el tipo de población a la que se dirige el proyecto Noria y el resto del trabajo de la brasileña: educación infantil y primaria.

Por último, todo conocedor de Lipman sabe que existe un tercer tipo de pensamiento que, en la década de los noventa, acabó de estructurar su modelo de pensamiento multidimensional: el pensamiento cuidadoso. Nuestro pensador integró cuatro modalidades de pensamiento bajo su paraguas: "appreciative thinking", "affective thinking", "active thinking", "normative thinking" y "empathic thinking" (LIPMAN, 2003: 264-270). El pensamiento cuidadoso entrena la sutileza para percibir ideas que escapaban a una aproximación analítica desprovista de vida. Así, la empatía con el otro y con la realidad, la reificación del pensamiento mediante la acción y la integración de las dimensiones afectivas abrían nuevas posibilidades que permitían un salto cuantitativo frente a un acercamiento basado exclusivamente en el pensamiento crítico.

\section{3. dimensiones experienciales de la filosofía. se abre el telón.}

El pensamiento cuidadoso servirá para enlazar la propuesta de Filosofía Experiencial con Niños/as. Lipman no sólo pretendió generar alumnos que pensasen mejor sino crear sociedades democráticas. A tal fin, era precisa una transformación de los participantes para que adquiriesen valores morales y de respeto mutuo. La consecución de esta metamorfosis no es sencilla cuando se trabaja con niños en riesgo de exclusión social y cuya identidad se ha forjado en escenarios experienciales teñidos por la violencia y los abusos. Nuestra hipótesis es que la transformación no depende, ni exclusiva ni principalmente, en la realización de actividades de análisis racional o de discusión grupales, puesto que 
la filosofía con niños como experiencia transformadora. una propuesta en organizaciones sin ánimo de lucro

ninguna de ellas ha sido la herramienta para la construcción de su identidad presente.

Hemos indicado las limitaciones de la aproximación analítico-discursiva en escritos anteriores (BARRIENTOS-RASTROJO, 2016); resumimos dos breves ejemplos por cuestiones de espacio. El primer problema es la disonancia cognitiva y consiste en que un cambio en ideas no siempre produce una modificación en nuestros sentimientos o reacciones ante la vida. Yalom ha narrado elocuentemente el caso en Quando Nietzsche chorou. Allí, Breuer, uno de los protagonistas, se queja de que por muy convincentes que resulten los argumentos de Epicteto (donde yo estoy no está la muerte y donde ella se encuentra, el sujeto desaparece) si reconocimiento o enunciación no produce los cambios que necesitaba en su vida para evitar su miedo a la muerte: "He aquí una verdad con una racionalidad suprema e irrefutable. Sin embargo, cuando esto verdaderamente asustado, ella no calma mis temores" (YALOM, 2007: 220). Idéntico es el caso de Pablo de Tarso cuando, en la Carta a los romanos, se lamentaba de que "No hago el bien que quiero, sino que hago el mal que no quiero" (Rm 7,19). El discípulo conocía la diferencia entre el bien y el mal, pero el acceso a las meras ideas no le permitía la transformación perseguida de su conducta.

La comprensión de la disonancia entre la vida (transformada) y la idea se produce desde varios esquemas; comenzaremos por dibujarla desde la perspectiva estética con Dewey. La experiencia no es una entidad que aparezca en áreas basadas en la razón analítica, no aparece "en el libro de contabilidad, ni tampoco en un tratado de economía o sociología o de psicología personal, sino en el drama o en la novela". El arte y las disciplinas vinculadas con la vida son aquellas que producen un golpe suficientemente sonoro para la metamorfosis. La experiencia "sólo puede ser expresada por el arte, porque ahí hay una unidad de experiencia que sólo puede ser expresada como una experiencia" (DEWEY 2008: 50). La experiencia no se consuma en el discurso, que es sólo una parte de ella, su exposición posterior, por el contrario, la experiencia es la realidad misma de la búsqueda y el impacto del viaje, de la incertidumbre y de la aceptación de la falibilidad y limitación humana. Reflexionar sobre la experiencia y hacer 
experiencia de una realidad son diferentes donde, la segunda es la determinante para la comprensión y transformación existencial. La comprensión de la muerte es la de quien ha estado a punto de fallecer siendo un complemento secundario la reflexión sobre la citada experiencia. Aquí empezamos a separarnos de Lipman, quien apuntó "Judgements are not ends in themselves. We do not experience works of art in order to judge them: we judge them in order to be able to have enriched aesthetic experiences" (LIPMAN, 2003:26). Volveremos a esto más adelante.

Para Dewey, la vida no es un trasunto de ideas sino de biografías. La biografía está formada no por los años vividos sino por el compendio de experiencias que nos han constituído. Por eso, las biografías dependen de lo que hemos experimentado y no de lo que pensamos, a menos de que el pensamiento sea experiencial. Aun en el último caso, el del pensamiento experiencial, se pone de manifiesto la recupera la centralidad de la experiencia: (1) ésta será crucial para la constitución del sujeto, (2) se concrete por medio de pensamientos experienciales, ejercicios experienciales o cualquier otra entidad teñida por ente adjetivo, que se hace sustantivo.

Por ello, nuestras decisiones parten de lo que somos (fruto de nuestra experiencia) antes que de lo que pensamos. Si esto es así, la configuración democrática a la que apela la Filosofía para/con Niños debería reconsiderar las metodologías de una racionalidad analítica y analizar las potencias experienciales de cada uno de sus elementos: tipos de palabras usadas, modos de escucha, formato del espacio y el tiempo que sean promotores de la experiencia, etc.

Un segundo argumento apoya la necesidad de un abordaje experiencial del trabajo filosófico: la distancia entre las ideas y las creencias. De acuerdo con Ortega y Gasset, tenemos ideas pero somos (o estamos) en nuestras creencias (ORTEGA Y GASSET, 1994a: 383). La posesión de las ideas implican que su naturaleza sea como la de un objeto que nos pertenece: podemos abandonarlas si se demuestre que eran erróneas. Por ejemplo, podemos pensar que el SIDA era una enfermedad incurable en los años ochenta, aunque la aparición de una vacuna modificaría nuestra idea sin grandes resistencias. El escenario muda si alguien 
la filosofía con niños como experiencia transformadora. una propuesta en organizaciones sin ánimo de lucro

intentase convertir a un ateo en creyente o viceversa, es decir, cambiar sus creencias. Cambiar nuestra idea sobre la curación del SIDA no va a modificar nuestra identidad (teniendo presente de que ni nosotros ni nadie querido está enfermo), un cambio en nuestra fe trastoca esencialmente lo que somos (tal como demostraron las grandes conversiones de la historia de la humanidad). Esta distancia entre un ejemplo y otro se explica con la frase del principio de este párrafo: "se tienen ideas pero somos (o estamos) nuestras creencias". La metamorfosis creencias muta lo que somos porque nosotros estamos en ellas, les pertenecemos. Si nosotros cambiamos nuestras ideas, son las creencias las que nos pueden cambiar. Estar en un contenedor nuevo de creencias nos convertirá en otros seres. He aquí el problema: ¿cómo modificar algo que es mayor que yo?

Resulta relativamente sencillo modificar las ideas de las personas mediante procesos de diálogo por la relación que mantienen con el sujeto, pero, y damos un paso más, siempre que sean coherentes con el contenedor creencial al que pertenecemos. Un católico no tendrá problema para aceptar la posibilidad de la curación de una enfermedad que consideraba incurable; pero mostrará resistencia a admitir que María, la madre de Jesucristo, no era virgen puesto que esa es una de las bases constitutivas de su fe. Su defensa es de tal índole que puede dar su vida frente a quien piense lo contrario.

El cambio del sistema de creencias requiere la muerte del propio yo. Si el sujeto está determinado por su fe, un cambio en el sistema de creencias sólo es posible si el individuo se transforma en otra persona o viceversa, el cambio de la persona en otro sujeto depende de su inserción en un sistema de creencias nuevo, pero, repitámoslo, sería la creencia la que, en última instancia, tiene poder para dar carta de libertad al sujeto para tal acto.

El impacto de una experiencia, con frecuencia inesperado, facilita el cambio. Imaginemos una persona cuyo sistema de creencias integra la idea del amor eterno y la imposibilidad de la infidelidad. Un día encuentra a su pareja en la cama con otra persona. Esa experiencia trastoca su sistema de creencias $\mathrm{y}$, por ende, su vida completa: a partir de ahora, manifiesta una sospecha inédita con sus compañeras/os, les exige sinceridad, realiza pruebas para verificar no caer 
nuevamente en la celada, manifiesta sentimientos de inseguridad nuevos y, obviamente, sus convicciones sobre el amor se modifica sustancialmente. Modificar las asunciones sobre la fidelidad con argumentos discursivos (ideas) antes de la traición sería inviable aunque se aportasen argumentos coherentes, consistentes, con bases suficientes, con clara conexión entre las partes y el todo, o entre las causas y los efectos, relevantes, claros y, en general, válidos. La persona podría aceptar la posibilidad de la infidelidad, pero no lo asumiría como una opción en su propia existencia. Análogamente, no asumiría el argumento de la fidelidad eterna tras su amarga experiencia. El caso es análogo en muchos votantes decepcionados con la política a principios del milenio en España que recuperaron la esperanza tras vivir la experiencia del 15-M.

Si la hipótesis es razonable, resulta crucial ubicar el concepto experiencial en el centro de la Filosofía con Niños, sobre todo, en aquellos casos donde la disonancia cognitiva es obvia. Su utilización no invalida el pensamiento creativo o crítico sino que, incentivando el cuidado por la reflexión, mejora la eficacia de ambos. Se puede ampliar la idea del concepto experiencia en escritos previos del autor (BARRIENTOS-RASTROJO, 2011; 2013)

\section{2. filosofía experiencial para niños/as.}

\section{1. bases i: experiencias.}

La Filosofía Experiencial para Niños obliga al facilitador a cuidar y diseñar tres elementos:

- La producción de ejericios con potencia experiencial.

- El fomento de disposiciones que faciliten la aparición de verdades experienciales.

- El establecimiento de escenarios experienciales.

Una experiencia es una vivencia que implica la reconfiguración identitaria del sujeto por medio del descubrimiento de evidencias que abren y cierran posibilidades existenciales previas. Lo explicamos por medio de un ejercicio experiencial propuesto por Walter Benjamin: 
la filosofía con niños como experiencia transformadora. una propuesta en organizaciones sin ánimo de lucro

En nuestros libros de cuentos está la fábula del anciano que en su lecho de muerte hace saber a sus hijos que en su viña hay un tesoro escondido. Sólo tienen que cavar. Cavaron, pero ni rastro del tesoro. Sin embargo, cuando llega el otoño, la viña aporta como ninguna otra en toda la región. Entonces, se dan cuenta de que el padre les legó una experiencia: la bendición no está en el oro sino en la laboriosidad (BENJAMIN, 1973: 167)

La actividad y la frustración de los hijos es la clave para un aprendizaje que modifica su esquema de pensamientos, de voluntades y de afectos, al menos en un sector de la realidad. A partir de entonces, entienden que lo importante no es el dinero sino el trabajo duro y esto determina una máxima que asumen como propia puesto que una de las caracTerísticas de este aprendizaje es que debe aprenderse por sí mismos.

Un segundo ejemplo clásico aparece cuando una persona se enfrenta a su muerte, base de la praemeditatio malorum de los estoicos. Imaginemos que a una empresaria de cuarenta años es diagnosticada con un cáncer de páncreas. El médico le advierte de que las posibilidades de recuperación son remotas, pero que hará todo lo posible por curarla. Ella inicia todos los procedimientos médicos oportunos, y, sobre todo, comienza el viaje de empezar a despedirse. La experiencia de este viaje la capacita con habilidades que le descubren realidades previamente desconocidas: el valor de cada día, la intensidad de la luz de un atardecer, el profundo significado de una mirada de apoyo, etc... Además, relativiza realidades que antes constituían la base de su jerarquía vital: lograr la presidencia de la empresa, imponer su propio criterio sobre el de los demás, sus enfados cuando las acciones no se cumplían milimétricamente, etc... El proceso cambia su forma de vida (sistema de creencias e identidad) y su reacción ante las circunstancias, no sólo por las restricciones que la enfermedad le impone (puesto que después de su curación las mantiene): cambia sus aspiraciones, genera otros deseos nuevos, inicia una nueva relación con el tiempo, etc... Concretando, cuando antes de su enfermedad alguien en su círculo le aconsejaba detenerse o disminuir la velocidad porque "estaba perdiéndose la vida auténtica", su sistema de creencias reaccionaba y generaba una mirada condescendiente respecto a su "supuesto" salvador. Si nos preguntamos cómo ha sido posible el cambio de creencias, una primera respuesta es clara: éste no sostiene la vida anterior por lo 
que asume su muerte $\mathrm{y}$, por ende, es factible la entrada en otro modelo. Se empiezan a crear disposiciones para el cambio, que resumiremos abajo.

Dentro de la Filosofía para Niños, Lipman ha puesto algún ejemplo experiencial básico (aunque no ha desarrollado las razones del cambio salvo a apelar a la necesidad de realizar alguna actividad significativa) como cuando recomienda el ejercicio de mantener su asombro y el deseo de encontrar soluciones en una clase a la que se muestra cómo el papel tornasol cambia debido a introducirlo en una solución básica o ácida (LIPMAN, 2003:275). La propuesta es cambiar una formación donde se explica al alumno la causa a otra en que descubra (o se viaje (KOHAN, 2015; LARROSA, 2014) por sí mismo las razones. El aprendizaje no sólo se centra en la respuesta sino en la adquisición de habilidades de indagación, de creatividad, de pensamiento crítico o de agudeza o cuidado que signarán su forma de ser en el futuro. Llama la atención que siendo tan trascendental este asunto para el aprendizaje y constitución del niño no existan más estudios profundos sobre el sentido de la experiencia (en el sentido de experiencia aquí usado, es decir, el trabajo directo con experiencias y no sólo la reflexión sobre ellas ${ }^{7}$ ), su diferencia con respecto a aprendizajes que no provocan estos cambios identitarios, el análisis de las disposiciones que facilitan la adquisición de las experiencias o las características (epistémicas, ontológicas, metafísicas, etc...) de los escenarios experienciales. Quizás, los más interesantes sean los de Larrosa, aunque se echa de menos una mayor incidencia en los aspectos filosóficos indicados y una propuesta práctica. También hay que apelar a el análisis de la condición filosófica de la experiencia en las sesiones realizadas por Kohan en Infancia y filosofía. Ni que decir tiene que el tema de su escrito es "¿qué torna filosófica una experiencia de pensamiento?" (KOHAN, 2009: 65) y aquí no interesa qué torna experiencial al acto filosófico.

En síntesis, una propuesta experiencial de la Filosofía con Niños supone centrarse en la realización de experiencias y, muy secundariamente, la reflexión sobre las mismas. Nótese el subrayado de secundariamente, puesto que esa

\footnotetext{
${ }^{7}$ La influencia del profesor Kohan sobre trabajos de investigación en Brasil ha provocado que este tema se abra como puede verse en los trabajos de la web nefiedicoes.filoeduc.org (último acceso, 28 de abril de 2019).
} 
la filosofía con niños como experiencia transformadora. una propuesta en organizaciones sin ánimo de lucro

reflexión, en muchos casos, ni siquiera sería necesario en la propuesta aquí esbozada. Imaginemos que deseamos que una adolescente entienda el mal que hace al golpear a un compañero. En varias ocasiones, se reúne con ella la directora del colegio, el jefe de estudios y el orientador del centro. Le han explicado (ideas) el daño que provoca, pero los resultados han sido infructuosos. Ella se ve empoderada por su grupo de extorsionadores y su actitud (sistema de creencias) se refuerza con la experiencia del maltrato de su padre sobre su madre. El estado conduce a la expulsión de la alumna, que entra en otro colegio. Allí, empieza a sufrir los mismos abusos que ella infringió. Entonces, comprende (experiencialmente) el daño que cometió sobre su compañera al morir las condiciones de posibilidad de despliegue de su anterior ser. Una reflexión sobre el hecho podría ser útil pero el acto hermenéutico no depende de ella porque la experiencia de sufrir en propia piel los sufrimientos infringidos es suficiente para lograr un aprendizaje que marque toda su existencia.

\section{2. bases ii: disposiciones.}

No todos los ejercicios o vivencias provocan cambios de la magnitud deseada. Ítem más, un mismo ejercicio puede ser iluminador para un sujeto e intrascendente para otro. De hecho, un niño en una sesión de filosofía donde se trabaje la justicia puede modificar sus patrones de conducta mientras que, a su compañero, la actividad puede resultarle "ridícula" o "aburrida".

La conversión es facilitada por las disposiciones para el cambio. El aburrimiento denota que la vivencia no ha mudado en experiencia. Si el ejercicio fue el mismo para ambos, ¿no deberíamos cuestionarnos si el fracaso del ejercicio se debió a que un niño cumplimenta disposiciones ausentes en el otro? Si la hipótesis es correcta un programa de filosofía experiencial con niños no sólo debería diseñar ejercicios sino trabajar disposiciones que incrementasen el potencial metamórfico de las actividades.

Las disposiciones consisten en las ubicaciones existenciales y subjetivas ante los ejercicios que aspirar a convertirse en experienciales. Conllevan el logro de las localizaciones adecuadas para incentivar los objetivos y la evitación de aquellas 
que las impiden. Por ejemplo, será útil promover una mente abierta ante los acontecimientos y sortear el cierre o la soberbia, puesto que la última impide aprehender los contenidos de verdad que contienen.

Hasta el momento, hemos detectado las disposiciones abajo indicadas (un listado no cerrado a futuras ampliaciones), que podrían completarse con algunas virtudes éticas y dianoéticas clásicas. Por razones de espacio, nos limitamos a citarlas y explicarlas brevemente aunque estamos preparando un libro donde todas serán adecuadamente descritas con referencias filosóficas completas:

(1) Arrojo frente a sucesos peligrosos.

Las Disertaciones por Arriano describen cómo el estoico ha de conducirse ante aquellos que amenazan nuestra propia integridad por medio de la muerte, el castigo o la esclavitud (ARRIANO, 1993: 58). Epicteto enseña que la máxima riqueza es la libertad del alma, la cual sólo puede ser esclavizada si el miedo la detiene, liberado de éste no hay posibilidad de sometimiento y el sujeto se arrojará a cualquier peligro. El control de la opiniones o de las representación de los hechos permitiría esta autonomía. Por ello, el trabajo con ejercicios estoicos puede ayudar a la apertura de la disposición experiencial, tal como hemos demostrado en una investigación empírica anterior que, actualmente, se encuentra en evaluación en una revista de Filosofía Aplicada española.

Una de las acepciones de la raíz de la experiencia, "per", se vincula con peligro (ORTEGA Y GASSET, 1994b: 175). La principal amenaza es la pérdida de la propia vida, pero esto es inherente a cualquier experiencia, tal como veíamos arriba. Ahora bien, ¿qué miedo habrá de tenerse si esta pérdida implica un acercamiento a una existencia más auténtica y más ensimismada frente a una insomne y que no pertenece al sujeto?

Disponerse con valentía ante lo inexplorado es una de las primeras características de quien quiere adquirir experiencia. El pusilánime se incapacita para adquirir la verdad que la entrada en un nuevo contenedor creencial le proporciona. 
la filosofía con niños como experiencia transformadora. una propuesta en organizaciones sin ánimo de lucro

(2) Coraje para atravesar puertas que no permiten el paso atrás.

Vinculado con lo anterior se encuentra el ánimo corajudo para mantener el arrojo. No es suficiente lanzarse al vacío sino perseverar en la decisión de no abrir el paracaídas a pesar del pánico cuando se cae en el abismo y ante la evidencia de la imposibilidad de volver atrás. De hecho, Claude Romano (2012) ha explicado la experiencia como un acto natalicio que no permite el regreso: nadie puede desnacer o regresar de la muerte. Entiéndase el nacimiento no sólo como el punto en el que iniciamos nuestra vida de carne y hueso sino como cada vez que se inicia una existencia con unas coordenadas nuevas que determinan nuevos modos de pensar, sentir o reaccionar afectivamente ante el mundo, es decir, cuando adquirimos una nueva identidad.

(3) Paciencia y dejar desplegar la experiencia.

El experimentado custodia y se hace acreedor de una relación con el tiempo específica: la demora. Ésta acarrea esperar sin esperanza, pero también permitir la apertura de cada ente real desde su legalidad temporal: dar tiempo a los entes con los que nos relacionamos. Decía Aranguren que "la experiencia de la vida requiere aguardar y tener paciencia, dar tiempo al tiempo; la experiencia de la vida tiene que sobrevenir no puede ser provocada o anticipada" (ARANGUREN, p. 34), puesto que esto es un ejemplo de obligar a la realidad a que se manifieste desde el propio esquema. Igual que no se puede obligar la flor del jazmín a desplegar su fragancia, la vida y la experiencia contienen tiempos a los que hemos de plegarnos si queremos que nos deleite con sus aromas y misterios.

Esta dinámica se aprecia en el arte: “Toda obra de arte posee una suerte de tiempo propio que se nos impone" advierte GADAMER (1994: 110) y Jauss avisaba de los riesgos de la precipitación y daba pautas pedagógicas para una captación adecuada:

Quien percibe estéticamente una pintura, es decir, quien quiere adquirir un nuevo conocimiento a través de la vista, ha de hacer frente a la inclinación de identificar o reconocer con precipitación $\mathrm{y}$, en vez de ello, ser consciente de cómo, para el contemplador, la significación - el objeto de la realidad figurada- se constituye poco a poco a partir de unas manchas de colores extrañas en un principio (JAUSS, 2002: 69) 
En este sentido, hay que dejar que la experiencia "siga su curso hasta su cumplimiento", así, el hecho no se consumirá sino que se consumará: "Una parte del trabajo se termina de un modo satisfactorio; un problema recibe su solución, un juego se ejecuta completamente; una situación, ya sea la de comer, jugar una partida de ajedrez, llevar una conversación, escribir un libro, o tomar parte en una campaña política, queda de tal modo rematada que su fin es una consumación, no un cese" (DEWEY, 2008: 41-42).

Esto exige el citado arte subjetivo de la demora, del retardo para dejar emerger los significados inherentes a la realidad" (ESQUIROL 2015: 49).

(4) Fascinación aperturista y seducción.

Junto al coraje y la audacia por la aventura, encontramos la seducción por lo desconocido. El abismo puede afrontarse como un monstruo paralizador o como un lugar desconocido al que aspirar. El primero es generador de la huida y, por ende, incapacita para descifrar su misterio. Por ello, aun cuando el abismo rompa los esquemas y esperanzas programados, debe mantenerse abierta la idea de que la sorpresa es bienvenida. Lo analítico se mueve por esquemas y programas donde todo está previsto, pero la vida recorre el camino de la sierpe, de la serpiente, siguiendo la terminología zambraniana, y la vida descubre sus profundidades por senderos inesperados. Al otro lado, el esquema determinado, determinante y determinador impide la innovación de la creatividad y la constitución del sujeto, puesto que, a medida que se descifra la realidad se forjan los métodos para su revelación.

El elemento determinador se antoja en el horizonte orwelliano de 1984 o en el foucaultiano de una sociedad que establece a sus consumidores (insomnes) del modo más adecuado a sus intereses. Si es esto es así, el cambio no sólo sería algo que nos debería fascinar sino que muta en el principio de nuestra liberación.

Mantenerse fascinado y seducido por lo inesperado es el principio para que la actividad experiencia rinda un nuevo ser en nosotros mismos y que la muerte del anterior no sea una catástrofe. 
la filosofía con niños como experiencia transformadora. una propuesta en organizaciones sin ánimo de lucro

(5) Compromiso con la autenticidad y la autocrítica.

No es legítima cualquier creación en el marco de la experiencia: no se trata de abogar por el pensamiento creativo sin dirección predeterminada o sin establecer una modalidad, puesto que esto despeñaría al sujeto en una alter-ación, desfocalización del yo y agonía en una alteridad indeterminada que no encuentra sosiego.

En consecuencia, el guía del camino es una autocrítica que eleva en niveles más profundos de autenticidad. Llama la atención que esto no es un egolatría sino un desasimiento del yo debido a la autocrítica, es decir, la afirmación de nuestra propia limitación para engrandecernos más allá de nosotros mismos. De esta forma, la llamada a la comunidad lipmaniana es un paso oportuno para, saliendo de uno mismo, alcanzar verdades que exceden la individualidad. Conocer las propias limitaciones y errores es más accesible para aquellos que nos ven desde su propia atalaya, diferente a la nuestra.

Sin embargo, la comunidad de investigación no es suficiente: se ha de alcanzar superar la visión del propio sujeto, aun cuando este se conceptúa como "comunidad". Así, hay que abrirse a un escenario experiencial, es decir, que sea ésta la que revele su esencia. La experiencia puede ser vivida por una persona pero es más que ella porque puede la comunidad puede hacer acopio de ella. Ahora bien, la experiencia es más que la comunidad porque, además, puede ser histórica (hay experiencias históricas) e incluso alcanzan niveles eidéticos, en el sentido platónicos y son un ente en sí mismo. Por ejemplo, el amor puede ser la experiencia que siento por mi pareja, la que siente mi familia por mí, la del mexicano por su patria, el amor cortés medieval o incluso la fuerza de atracción ontológica citado por Scheler en su Ordo Amoris.

(6) Humildad.

La minoración del individuo deriva de su humildad: "La experiencia es experiencia de la finitud humana" señala Gadamer (2000: 433). El progresivo debilitamiento y deslocalización del sujeto es proporcional a su integración en experiencias que lo transcienden. 
La posibilidad de que el adolescente acepte que su idea de justicia sea una posible entre muchas funda el hecho de que entre en su realidad una visión más plural del concepto y que escape del provincianismo intelectual de su concepción de justicia. No sólo aceptan nuevas ideas de la justicia sino una experiencia de la misma más rica sin que esto implique que ninguna de las dos acepciones sea falsa. Expliquémoslo con un ejemplo musical: la quinta sinfonía de Beethoven puede interpretarse con un único instrumento o con una orquesta completa. En los dos casos, la sinfonía es la misma partitura pero la segunda permite apreciar matices inéditos que la interpretación de un violín solitario no es capaz de exponer aisladamente: ese instrumento eremita no conseguirá reproducir los sones abisales de la percusión o la dulzura de ciertas notas que nos lega la flauta.

La humildad permite al violín asociarse con la orquesta $\mathrm{y}$, negándose engrandecerse en el conjunto: ser menos para convertirse en más.

Al otro lado de la humildad, la soberbia impide ver la verdad en los ejercicios experienciales. Si un sujeto se conduce a un taller asumiendo que posee todo el saber, su actitud lo incapacita para poder escuchar al resto y, por ende, para poder aprovechar el conocimiento de "lo otro".

(7) Equilibrio.

Desde el Tao ("las puertas desde el mundo material al mundo oculto se abren con la observancia de la tranquilidad" (ANTONOV, 2008: 7)) a Hume ("una perfecta serenidad mental, ciertos recuerdos, una atención apropiada al objeto: si faltara cualquiera de estas circunstancias, nuestra experiencia sería engañosa y seríamos incapaces de juzgar la belleza con alcance universal" (HUME, 1989: 31)) se incentiva el equilibrio como notación específica para la adquisición de las verdades profundas.

La experiencia requiere estar focalizado en el objeto, no tener la atención requerida por múltiples instancias, demorarse en el objeto para no quedar alterado (ORTEGA Y GASSET, 1994a: 299), en otro (alter), sino en uno mismo.

Por ejemplo, el trastorno a que aboca la ira hurta la atención y obnubila el juicio, impidiendo el nacimiento de la evidencia, de la verdad experiencial. De ahí, la importancia de que una sesión de Filosofía Experiencial comience por un 
la filosofía con niños como experiencia transformadora. una propuesta en organizaciones sin ánimo de lucro

ejercicio de focalización en el instante presente o que la prosoche sea uno de los ejercicios básicos con que se entrenaban los estoicos (y, por tanto, base de un proyecto de talleres experienciales, como veremos más adelante).

(8) Vocación de retiro.

Lo anterior explica la vocación de retiro de la experiencia (muy diferente al aislamiento). El retiro no conmina a apartarse del mundo como un eremita (Comte-SPONVILLE, 2000: 29; MARÍAS en AAVV, 1966: 119) sino que las acciones estés centradas, que partan de un centro no alterado.

(9) Búsqueda minuciosa y sutil usando los modelos artístico-estéticos (el gusto), espirituales y sapienciales.

La sutileza es la capacidad de escuchar o ver realidades inaccesibles a aquel que no posee preparación suficiente. Según el Tao, “la persona sabia puede ver lo invisible para un ojo normal y conoce las cosas que no pueden ser alcanzadas ni caminando ni montando" (ANTONOV, 2008: 28). Esto no es una potencia vinculada exclusivamente con la religión, pues, como explica William James "se ha de tener oído musical para saber el valor de una sinfonía, se ha de haber estado enamorado para comprender el talante anímico de un enamorado. Si nos falta el corazón o el oído, no podemos interpretar justamente al músico o al amante e incluso podemos considerarlo absurdo o menguado mental" (James, 1994: 179).

Los escritos sapienciales, los espirituales y los estéticos ofrecen, entre otros, mecanismos para ir adquiriendo una mejora en la habilidad para intuir lo que no está a disposición del común de los mortales $^{8}$. Disponerse a buscar en lo pequeño es el primer paso. Para ello, hay que permitir a la realidad que nos impacte; en palabras de Heidegger: “Hacer experiencia con algo (...) quiere decir: dejarlo venir a nosotros (...), que nos alcance, se abalance sobre nosotros, nos dé la vuelta y nos transforme" (HEIDEGGER citado en ROMANO, 2012: 212)

\footnotetext{
${ }^{8} \mathrm{Ni}$ que decir tiene que estos escritos también podrían acarrear consecuencias dogmatizantes y doctrinarias en los sujetos. Sin embargo, esto vulneraría su naturaleza aperturista como explicamos en un artículo reciente (BARRIENTOS-RASTROJO, 2018).
} 


\section{3. escenarios experienciales.}

Si las disposiciones dibujan las ubicaciones subjetivas que incrementan las posibilidades de la iluminación experiencial, los escenarios constituyen los contextos del ejercicio, es decir, la cualificación de los marcos en que se producen. Por ejemplo, cabe considerar como escenario temporal una cronología instrumental que no buscase un fin sino el consumo del tiempo, pero para la experiencialidad es más acorde un tiempo consumatorio, es decir, uno que extraiga de éste todas sus posibilidades. Por otro lado, el escenario epistemológico determinará el tipo de verdades de la experiencia: mera información o contenidos que no provoquen una mutación en la persona o la evidencia, es decir, un contenido informativo menor aunque con mayor capacidad metamórfica. Veamos cinco escenarios experienciales como muestra.

(1) Escenario temporal: el viaje y la consumación.

La palabra alemana que apela a la experiencia es Erfahrung y su verbo fahren significa viajar (ORTEGA Y GASSET, 1994b: 176). Asimismo, la raíz del término ("per") se vincula con "peiro", que expresa, en griego, cruzar; "perao" es "pasar a través de"; "peraino" es "atravesar hasta el borde", puesto que el límite en griego es "perás"; finalmente, el viajero es el "peratés" (ROMANO 2012: 213), aunque también puede ser un "pirata", alguien que recorre la vida sin miedo a la muerte (ANDERS, 2001-2009)

Estos viajes tienen poco que ver con los desplazamientos que hoy pues su fin no es interno a ellos mismos sino que responden a un fin externo. El viaje posee valor en sí mismo, es la base de la transformación, y, por ende, cada una de sus etapas ha de vivirse intensamente. Ésta es la razón de que Kavafis indicase que el viaje fuera largo y lleno de aventuras. Algo que no concierta con el intento actual de tener viajes cada vez más rápidos y con menos riesgos.

El viaje experiencial consiste en "caminar cuando no había caminos, sino que todo viaje era más o menos desconocido y peligroso. Era el viajar por tierras sin guía previa" (ORTEGA Y GASSET, 1994b: 176), pues obligada a crear un camino (y un rostro) propio por el que, luego, otros podían transitar. 
la filosofía con niños como experiencia transformadora. una propuesta en organizaciones sin ánimo de lucro

En el plano experiencial, no se trata de que se cree un camino sino de que uno mismo se convierte en camino y en guía del sendero que él mismo construyó. Se reitera la idea machadiana: "caminante no hay camino, se hace camino al andar". El saber experiencial responde a este modelo: al del gran artista que, luego, es copiado por los discípulos 9; el del gran filósofo, como Platón, que, años después, es seguido en la Academia. Ahora bien, el discípulo puede acabar iniciando su propio camino y hacerse maestro, como en el caso de Aristóteles respecto al citado Platón o los estoicos respecto a Aristóteles y así sucesivamente.

El viaje como escenario temporal obliga a conectar realidades de aquel con lo sucedido en las sesiones filosóficas. He ahí el sentido de explicar en el contexto de los talleres de ítems como el cansancio, los senderos que se abren en dos, los salteadores, las tormentas, la nostalgia, el fin, las piedras, los días lluviosos y los agostados, las fragancias y el hedor, el sudor, la visión de la puesta de sol, la culminación de una meseta, los desfiladeros o las caminadas en círculo.

Por otro lado, la relación con el tiempo no puede ser la de la consumición sino la de la consumación. No hay que "matar el tiempo" con actividades sino realizar ejercicios que lo eleven, que le devuelvan un brillo que lo haga eterno, es decir, que se mantenga siempre en el recuerdo que recuperen su sentido siendo conscientes de su limitación. Esto se traslada al campo biográfico: hay que mudar la existencia desde un plano instrumental y carente de sentido hacia una progresiva valoración de su entraña. Se trata de saltar desde la consideración de una graduación académica como "acto social" a la de cumplimentación de una etapa vital propia de alguien cercano. Si se consigue percibir las dimensiones trascendentales del hecho, el tiempo pasa de sus connotaciones cronológicas a las kairóticas, el hecho se convierte en un símbolo que acaba constituyendo al sujeto y en un recuerdo inmarcesible que dota de un valor especial no sólo a ese momento sino a su vida (BARRIENTOS-RASTROJO, 2015). Esta relación temporal debería

\footnotetext{
9 "El artista tiene que ser un experimentador, porque tiene que expresar una experiencia intensamente individual a través de medios y materiales que pertenecen al mundo común y público (...). Si en vez de decir "experimental" dijéramos -aventurero- probablemente obtendríamos la aceptación general, tan grande es el poder de las palabras. Porque el artista es un amante de la experiencia sin escoria, evita los objetos ya saturados y está, por consiguiente, siempre a la última. Por naturaleza, es un insatisfecho de lo establecido como lo es un explorador geográfico (DEWEY, 2008: 162).
} 
buscarse también en el trato con la palabra (hasta alcanzar una palabra diciente en el sentido gadameriano) y con los sucesos de los talleres filosóficos.

(2) Escenario epistemológico: osmosis (morfología metodológica) y evidencia (producto).

El aprendizaje experiencial no se produce de forma secuencial, es decir, como en una cadena de montaje; por el contrario, el proceso es osmótico, va calando de fuera hacia adentro y por todos los flancos de la membrana existencial.

Como en una canción, la persona puede mostrar resistencia inicialmente, aunque el impacto inicial le obliga a seguir escuchando hasta que queda atrapada: se va haciendo dueña hasta que la persona sólo vive por medio de la música (de ahí la disposición de la seducción), siguiendo el modelo del genitivo subjetivo que veremos a continuación.

El impacto es análogo al de esa pintura que, en medio de los millares de lienzos de un museo, nos cautiva por senderos que, inicialmente, no atisbamos siquiera a comprender. De hecho, la sensación inicial no es necesariamente la de atracción sino, quizás, la de desconcierto. El individuo puede revelarse y afirmar, con Zambrano, que la verdad que nos traslada es "terriblemente pobre en contenido intelectual"; sin embargo, progresivamente, se percata de que "opera en la vida una transformación sin igual que otros pensamiento más ricos y complicados no fueron capaces de hacer" (ZAMBRANO, 1995: 69). He aquí el eje de la evidencia, la verdad propia de la experiencia. El misterio de la experiencia no es extensiva sino intensiva, no opera en horizontal sino en línea vertical: “se trata de redescubrimiento. No es una verdad nueva, sino una forma que toma algo que ya se sabía, y que ahora penetra en la vida moldeándola; es algo que antes no operaba y que ahora se ha vuelto operante" (ZAMBRANO, 1995: 69).

(3) Escenario hermenéutico: anagogía.

La epistemología evidencial se conecta con la anagogía. Ésta implica una coincidencia entre "los modi essendi, los modi cognoscendi y los modi interpretandi" (ANTÓN PACHECO, 2010: 151), es decir, conocer algo implica una transformación esencial del sujeto puesto que hay una coherencia entre los plexos intelectivos, los interpretativos o, en general, cualquiera de los del ser. Cuando 
la filosofía con niños como experiencia transformadora. una propuesta en organizaciones sin ánimo de lucro

una mujer tiene a su primera criatura no sólo se transforma el conocimiento de la expresión "ser madre" sino que se convierte en madre, pasa de ser hija o esposa a adquirir una existencia esencial nueva que trastoca todas sus dimensiones.

Lo mismo sucede con el místico: no se caracteriza por poseer un conocimiento intelectual superior al del ateo sino por encarnar a dios en este mundo. Sin esa encarnación, no alcanza su saber y, viceversa, la encarnación implica de modo irremisible el conocimiento de Dios en los niveles sugeridos. San Bernardo ha retomado el concepto de participación de Platón para señalar que sólo lo semejante puede hablar de lo semejante (TORRE, 1987: 18). Sólo siendo como Dios se puede hablar adecuadamente de él.

Desde la perspectiva de una filosofía experiencial, el ejercicio es esencial en la medida en que busca la equiparación con la raíz de la experiencia que se busca. Kitaro Nishida caracteriza la experiencia como el momento en que no hay “separación de conocimiento, sentimiento y volición" (NISHIDA, 1995: 90). Añade que, "en el momento de la experiencia pura, no hay todavía ninguna oposición de sujeto y objeto (...) solo existe una actividad pura, independiente, autosuficiente" (NISHIDA, 1995: 90). Esto nos conduce al último escenario que explica por qué el acto filosófico experiencial no es cognitivo, emocional o volitivo sino una acción que trasciende la limitación individual y se convierte en un acto ontológicoexperiencial por medio del genitivo subjetivo.

(4) Escenario ontológico: genitivo subjetivo.

El término genitivo subjetivo procede de Panikkar aunque el concepto sea muy anterior. Él distingue el genitivo objetivo del genitivo subjetivo como sigue: "no es mi experiencia sobre Dios, sino la experiencia de Dios -en mí y a través de mí- y de la cual yo soy consciente" (PANIKKAR, 2001: 78).

La filosofía experiencial se analiza de modo autocrítico para alcanzar la comunidad, pero no queda en la comunidad presente sino que busca espacios o tiempos distantes. Como si de reducciones fenomenológicas se tratase, el sujeto seguirá alzándose por dos caminos: la disminución del yo y la ampliación de la experiencia en su seno, al punto de que el yo sólo se manifieste como exposición de la experiencia. 
Imaginemos que trabajamos la "fortaleza ante la traición". Las primeras etapas suponen el análisis personal del término. Seguidamente, se confrontará con la comunidad. Luego, el concepto habrá de entenderse más allá de lo que la comunidad conceptúe. Cada fase nos habrá llevado desde la visión discursiva del término a la vital por medio de ejercicios experienciales. Las fases más avanzadas suponen sentirse traspasado por el término para comprender anagógicamente sus consecuencias en la propia existencia.

Desde estas coyunturas, se entiende que el trabajo filosófico no apela a una comprensión discursiva del término sino a una transformación del sujeto. Siendo así, el escenario supera las dimensiones del pensamiento crítico o creativo puesto que ambos parten de ideas de sujetos. Por el contrario, este escenario pretende lograr que no sea el sujeto el que piense sino que éste acabe siendo pensado por medio de la experiencia perseguida, en nuestro caso, “la fortaleza ante la traición”. El yo se convierte en un caso más de esa experiencia y, por tanto, su excelencia hermenéutica depende de la anulación progresiva de la particular perspectiva de su acercamiento.

Por ello, "hacer experiencia con algo (...) quiere decir: dejarlo venir a nosotros (...), que nos alcance, se abalance sobre nosotros, nos dé la vuelta y nos transforme" (HEIDEGGER citado en ROMANO, 2012: 212).

Nótese que esto no disminuye a la persona sino que la incrementa e intensifica. Como señala Romano: "no es el hombre el que tiene una experiencia, es la ex-per-iencia la que hace al hombre. Únicamente en ella y por ella, accede el hombre a su humanidad" (ROMANO, 2012: 230).

Un modelo adecuado para esto sería un taller de índole estoica cuyas bases explicamos en un breve anexo al final de este trabajo, cerrando aquí la exposición por motivos de espacio.

\section{Agradecimientos}

El autor desea agradecer enfáticamente a los dos evaluadores ciegos del artículo por el conjunto de correcciones y sugerencias que han servido para mejorar este trabajo y para abrir vías de reflexión e investigación futura al autor. 
la filosofía con niños como experiencia transformadora. una propuesta en organizaciones sin ánimo de lucro

\section{bibliografía}

AUTORES VARIOS, Experiencia de la vida, Alianza, Madrid, 1966.

ANDERS, V. (ed.): Etimologías de Chile, Santiago de Chile, 2001-2019, recuperado de http:/ / etimologias.dechile.net/PIE/?per-5, último acceso 27 de abril de 2009.

ANTONOV, V. (ed.): Tao Te Ching, Lulú, Ontario, 2008.

ANTÓN PACHECO, J.A.: El ser y los símbolos, Mandala, Madrid, 2010.

ARRIANO: Disertaciones por Arriano, Gredos, Madrid, 1993.

BARRIENTOS-RASTROJO, J.: “Abrir preguntas esenciales como quehacer del maestro. De la quiebra de la pedagogía bancaria de Freire a la pregunta esencial gadameriana y al saber de la experiencia zambraniana", Diálogo filosófico, 2013(86), pp. 325-352.

BARRIENTOS-RASTROJO, J.: "An Experience workshop with groups. Theory and practice" en Weiss, M.N. (ed). The Socratic handbook, Lit Verlag, Zürich, 2015, pp. 375-383.

BARRIENTOS-RASTROJO, J.: "El rostro de la experiencia desde la marea orteguiana y zambraniana", Revista Endoxa, revista de la Facultad de Filosofía de la UNED, 2010, número 25, pp. 279-314.

BARRIENTOS-RASTROJO, J.: “La experiencialidad como respuesta a la tendencia analítica de la filosofía para niños", ChildhoodEPhilosophy, 12 (25), 2016, pp. 519-542.

BARRIENTOS-RASTROJO, J.: "La fisiología del saber de la experiencia y los frutos de su posesión", Themata, número 44, 2011, pp. 79-96.

BARRIENTOS-RASTROJO, J.: “La obcecación creencial y su tratamiento por medio de la comun(ica)ción experiencial y de la palabra invocadora", Ilu, 23, 2018, pp. 13-30.

BARRIENTOS-RASTROJO, J.: Los símbolos desfallecidos, Analogía Filosófica, número especial 36, México DF, 2015.

CARRERAS, C.: "Filosofía para Niños: el desarrollo global de la habilidades de pensamiento" en Barrientos-Rastrojo, J.: Filosofía para niños y capacitación democrática freiriana, Liber Factory, Madrid, 2013, pp. 91-113.

COMTE-SPONVILLE, A.: El amor. La soledad, Paidós, Barcelona, 2000.

BENJAMIN, W.: Discursos interrumpidos I, Taurus, Madrid, 1973.

BRENIFIER, O.: Filosofar como Sócrates, Diálogo, Valencia, 2011

BRENIFIER, O.: La práctica de la filosofía en la escuela primaria, Diálogo, Valencia, 2012.

DE PUIG, I. - SÁTIRO, A.: Jugar a pensar. Recursos para aprender a pensar en educación infantil (4-5 años), Octaedro-SEP, Puebla, 2008.

DEWEY, J.: Arte como experiencia, Paidós, Barcelona, 2008.

ECHEVERRÍA, E.: Filosofía para niños, SM, México DF, 2006.

ESQUIROL, J.M.: La resistencia intima, Acantilado, Barcelona, 2015.

GADAMER, H.G.: La actualidad de lo bello,Paidós, Barcelona, 1991.

GADAMER, H.G.: Verdad y método I, Sígueme, Salamanca, 2000.

HUME, D.: La norma del gusto y otros ensayos, Ediciones 69, Barcelona, 1989.

JAMES, W.: Las James, W.: Las variedades de la experiencia religiosa, Península, Barcelona, 1994.

JAUSS, H.R.: Pequeña apología de la experiencia estética, Paidós, Barcelona, 2002.

KOHAN, W.: El maestro inventor, Ediciones del Solar, Caracas, 2016.

KOHAN, W. Infancia y filosofía, Progreso, México DF, 2009.

KOHAN, W. Viajar para vivir: ensayar, Dávila, Buenos Aires, 2015.

KOHAN, W. - WAKSMAN, V.: Filosofía para Niños. Aportes para el trabajo en clase, Noveduc, Buenos Aires, 2000.

LARROSA, J.: Tremores, Autêntica, Belo Horizonte, 2014.

LIPMAN, M.: Philosophy goes to school, Temple University Press, Philadelphia, 1988.

LIPMAN, M.: Thinking in education, Cambridge University Press, Cambridge, 2003. 
LIPMAN, M. - SHARP, A. - OSCANYAN, F.S.: La filosofía en el aula, Ediciones de la Torre, Madrid, 1998.

MENDONÇA, D.: Brincar a pensar. Manual de filosofía para crianças, Plátano, Lisboa, 2011.

NISHIDA, K.: Indagación sobre el bien, Gedisa, Madrid, 1995.

ORTEGA Y GASSET, J.: Obras completas 5, Alianza, Madrid, 1994a.

ORTEGA Y GASSET, J.: Obras completas 8, Alianza, Madrid, 1994b.

PANIKKAR, R.: Iconos del misterio. La experiencia de Dios, Península, Barcelona, 2001.

ROMANO, C.: El acontecimiento y el mundo, Sígueme, Salamanca, 2012.

SÁNCHEZ ALCÓN, J.M.: Pensamiento libre para personas con discapacidad intelectual, Pirámide, Madrid, 2011.

TORRE, J.M de la: "Experiencia cristiana y expresión estética en los sermones sobre el Cantar de los cantares" en San Bernardo: Obras completas de San Bernardo V. Sermones sobre el Cantar de los cantares, BAC, Madrid, 1987, pp. 3-75.

YALOM, I.: Quando Nietzsche Chorou, Saída de Emergencia, Parede, 2007.

ZAMBRANO, M.: La confesión: género literario, Siruela, Madrid, 1995.

\section{Anexo. Una propuesta estoica con ONGs.}

A partir de la teoría expuesta, se pueden proponer diversas técnicas o programas experienciales completos. Uno de ellos es la base del Proyecto de Filosofía Aplicada Boecio. Actualmente, lo adaptamos como propuesta para el proyecto Maparra de Caritas donde hemos podido actuar gracias a la Asociación Tiritas Creativas.

Su objetivo consiste en "Entrenar-formar personalmente y desde la práctica a grupos de jóvenes y adolescente para que logren un mayor autoconocimiento, gobierno de las emociones y de la propia existencia y el pensamiento crítico usando como base la metáfora del entrenamiento personal y como medio de ejercicios de tradiciones sapienciales de la filosofía".

El público al que se dirige son niños y adolescentes en riesgo de exclusión social que han vivido situaciones complicadas como malos tratos, pobreza o dificultades sociales y deficiencias educativas.

El escenario del viaje se ubica en la imagen de la preparación de un marathon filosófico. Los participantes habrán de entrenar sus capacidades filosóficas para ser capaces para enfrentarse a las grandes pruebas dela vida que los desequilibran.

Los escenarios anagógicos se trabajan mediante la realización de ejercicios estoicos que ponen de manifiesto que el avance no reside en la memorización de textos sino en su articulación en la propia existencia.

De hecho, el escenario del genitivo subjetivo depende de la progresiva convicción de que no son ellos con sus dificultades los que han de vivir sino su regente interior, su gladiador profundo que los hace enfrentarse a los obstáculos del día a día. No serán ellos los que viven sino el ideal estoico a través de ellos. Así, cuando tomen una decisión no se preguntarán a ellos mismos sino a su profundo sentido interior que les determinará qué es lo correcto, tal como sucedía en el mundo estoico.

Las disposiciones se trabajarán transversalmente y mediante ejercicios particulares. Así, la sesión décimo cuarta implica un ejercicio donde se analiza todo aquello que hay dentro de una habitación mediante visiones parciales. Se incentiva así la agudeza y sutilidad para ampliar la visión. Otros ejercicios semejantes se realizan con otros sentidos como el olfato o el gusto. Asimismo, se afina la sensibilidad emocional con la contemplación de obras de arte.

Los contenidos se reparten en dos bloques que respetan las tres líneas estoicas: logos o pensamiento crítico, ethos o comportamiento y physis o ubicación cósmica en la totalidad.

Bloque I. Gobierno de las emociones (ethos) y localización existencial (physis)

(1) La filosofía como entrenamiento para la vida (sesiones 1-3). 
la filosofía con niños como experiencia transformadora. una propuesta en organizaciones sin ánimo de lucro

Se introducirá a los alumnos en el modelo de filosofía como camino hacia la preparación de una maratón existencia y como camino de vida. Asimismo, se abordará las ideas de los estoicos básicas y se empezará a trabajar el juramento del filósofo.

(2) Gobierno de las emociones (sesiones 4-5).

Se abordará la identificaciones de emociones descontroladas (pasiones), se ayudará a conocerlas, comprenderlas y la realización de ejercicios para gobernarlas.

(3) Presentación y representación (sesiones 6-7).

Se aprenderá a identificar aquello que el alumno puede cambiar de sí mismo y lo que no es posible. Asimismo, se incentivará la diferencia entre el hecho y la representación, enseñando cómo son éstas últimas las que determinan nuestras acciones y reacciones.

(4) Aceptación, humidad y escucha (sesiones 8-9).

Descubrir los elementos que no se pueden cambiar. Trabajar la aceptación de lo que no se puede cambiar. Desarrollar la escucha y la autocrítica.

(5) Caídas (sesiones 10-11).

Entrenar el momento de caída o los retrasos. Usar la caída como recurso para el avance.

(6) Desensibilización óntica o visión cósmica (sesiones 12-13).

Desarrollo del punto de vista cósmico usando textos de Marco Aurelio y Epicteto. Asimismo, se trabajará a partir del concepto de pre-juicio de Gadamer o afección en Marinoff.

(7) Atención plena o prosoche (sesiones 14-15).

Entrenamiento para focalizar en torno a lo minúsculo. La contemplación de lo pequeño y de la belleza. Habitar realidades invisibles para la gente vulgar.

(8) Praemeditatio malorum y habitando el dolor (sesiones 16-17).

Entrenar ante las imágenes de lo doloroso. Fortalecimiento antes las dificultades existenciales. Valorar lo que se posee desde la imaginación de su pérdida.

(9) Ejercitando en el límite (sesión 18)

Recapitulación de la preparación y enfrentamiento con situaciones complejas.

Bloque II. Pensamiento crítico (logos).

Este bloque desarrollará el pensamiento crítico usando ejemplos prácticos de la realidad social de los alumnos y a partir de diálogos basados en experiencias incentivando ejercicios reales entre las sesiones y dentro de ellas.

(10) Tipos de afirmaciones y estructuras de argumentos (sesión 19).

(11) Estructura de argumentos (sesión 20).

(12) Falacias y errores de pensamiento (sesión 21).

(13) Análisis del lenguaje (sesión 22).

(14) Pensamiento Crítico: Criterios de verdad (sesión 23).

recibido en: 28.04 .2019

aceptado en: 30.05 .2019 